\title{
Improved T-S Fuzzy Control for Uncertain Time-Delay Coronary Artery System
}

\author{
Zhenyu Zhu (D), Zhanshan Zhao $\left(\mathbb{D}\right.$, Haoliang Cui, and Fengdong Shi ${ }^{(D)}$ \\ School of Computer Science \& Technology, Tianjin Polytechnic University, Tianjin 300387, China \\ Correspondence should be addressed to Zhanshan Zhao; zhzhsh127@163.com
}

Received 8 March 2019; Revised 22 April 2019; Accepted 24 April 2019; Published 16 May 2019

Academic Editor: Yong Xu

Copyright (c) 2019 Zhenyu Zhu et al. This is an open access article distributed under the Creative Commons Attribution License, which permits unrestricted use, distribution, and reproduction in any medium, provided the original work is properly cited.

\begin{abstract}
This paper is based on the Takagi-Sugeno (T-S) fuzzy models to construct a coronary artery system (CAS) T-S fuzzy controller and considers the uncertainties of system state parameters in CAS. We propose the fuzzy model of CAS with uncertainties. By using T-S fuzzy model of CAS and the use of parallel distributed compensation (PDC) concept, the same fuzzy set is assigned to T-S fuzzy controller. Based on this, a PDC controller whose fuzzy rules correspond to the fuzzy model is designed. By constructing a suitable Lyapunov-Krasovskii function (LKF), the stability conditions of the linear matrix inequality (LMI) are exported. Simulation results show that the method proposed in this paper is correct and effective and has certain practical significance.
\end{abstract}

\section{Introduction}

Since Pecora and Caroll proposed chaotic synchronization in 1990 [1], synchronization control of chaotic systems has developed rapidly and has become one of the most important topic during the past 30 years [2-12]. Two chaotic systems synchronization of the main idea is to design a suitable controller, to make the state of the slave system track the status of the master system. In order to solve the synchronization problem of chaotic systems, many methods have been proposed, such as feedback control method [13, $14]$, continuous sliding mode method $[15,16]$, and fuzzy control method [17, 18]. Among them, the fuzzy control has attracted much attention because of its wide application and independent of the precise mathematical model of the controlled object and so on. Takagi-Sugeno (T-S) fuzzy model can provide the approximation of nonlinear features by fuzzy mixing of multiple local linear models with appropriate membership functions, by using fuzzy rules, the dynamic nonlinear systems are approximated to the set of the local linear input and output relation, and the whole fuzzy model is finally obtained by smoothing the set of the local linear model with the fuzzy piecewise membership function. In [19], the chaotic system is modeled as a T-S fuzzy system, and stable results are obtained, so it has been shown that the fuzzy control may provide a system effective frame for the nonlinear system control design. According to literature [20], we know that T-S fuzzy model has made remarkable achievements in solving complex nonlinear systems.

The vasospasms are the main cause of cardiocerebral vascular diseases. Once the blood vessels with the chaotic state, it will lead to the occurrence of cardiocerebral vascular diseases such as vasospasm. The main chaotic phenomenon of coronary artery is vasospasm. In order to understand the nonlinear characteristics of vasospasm and to control the occurrence of chaos, literature [21] proves that diseased vessels have the same behavior as normal vessels. In literature [22], a chaos suppression controller is designed based on variable structure control theory. Application of higher-order sliding mode adaptive controller is in reference [23]. They use a synchronization controller to synchronize the chaotic motion state of the diseased vessels to that of the normal vessels, so as to achieve the goal of treatment. However, there are some clinical factors, such as the effect of the body's own time delay on drug absorption in patients with coronary artery disease after taking the drug, not been proposed in the literature [21-23].

In order to reduce the negative impact of time delay, we need to reduce the conservatism of the system and improve the performance of the system; many researchers 
have used the triple integral form of LKF to analyze the stability of various time-delay systems in recent years, such as Wirtinger-based integral inequality [24], Jensen's inequality [25], and Wirtinger-based double integral inequality [26], and explain its effectiveness in reducing the conservativeness of stability criteria. Due to the method of literature [26] can be applied directly in finding lower bound of double integral, such as $\int_{t-\tau}^{t} \int_{s}^{t} \dot{x}^{T}(u) N \dot{x}(u) d u d s(N>0)$, and the method of literature [24] can not be applied directly in solving it. Therefore, literature [26] is more conservative than literature [24] when dealing with system stability analysis. In order to further reduce the conservativeness of system stability analysis, we study the stability of chaotic time-delay synchronous control systems by using the method in [26]. In addition, for solving complex nonlinear systems, we design a parallel distributed compensation (PDC) controller based on fuzzy model.

From the above discussion, this paper is to explore chaotic synchronization of uncertain CAS based on fuzzy models. A PDC synchronization controller for a class of uncertain CAS with time delay is proposed using T-S fuzzy. Using Wirtinger-based double integral inequality [26] and Moon et al.s inequality [27] can further reduce the conservativeness of CAS. It is show that the synchronization controller can synchronize the response system with the drive system, and the error system tends to zero.

The structure of the paper is as follows. Section 2 shows that the T-S fuzzy control problem formulation of the same class for time-delay uncertain CAS. Section 3 describes the design of T-S fuzzy synchronization controller. We design LKF and we can get the LMI by using the lemma mentioned in Section 4. Results of simulation are analyzed shown in Section 5. Finally, we will give a conclusion in Section 6.

\section{System Description and Problem Analysis}

In this paper, considering the normal uncertain CAS with time delay as the driving system, the rules of T-S fuzzy model can be described as follows.

Rule I

$$
\begin{aligned}
& \text { IF } x_{1}(t) \text { is } M_{l 1} \\
& \text { THEN } \dot{x}_{m}(t)=\left(A_{1}+\Delta A\right) x_{m}(t) \\
& \quad+\left(B_{1}+\Delta B\right) x_{m}(t-h(t))+E \cos (t)
\end{aligned}
$$

Rule II

$$
\begin{aligned}
& \text { IF } x_{2}(t) \text { is } M_{l 2} \\
& \text { THEN } \dot{x}_{m}(t)=\left(A_{2}+\Delta A\right) x_{m}(t) \\
& \quad+\left(B_{2}+\Delta B\right) x_{m}(t-h(t))+E \cos (t)
\end{aligned}
$$

Rule $i$

$$
\begin{aligned}
& \text { IF } x_{i}(t) \text { is } M_{l i} \\
& \text { THEN } \dot{x}_{m}(t)=\left(A_{i}+\Delta A\right) x_{m}(t) \\
& \quad+\left(B_{i}+\Delta B\right) x_{m}(t-h(t))+E \cos (t)
\end{aligned}
$$

where $x_{m}(t)$ is a state vector for driving system, $M_{l i}(l=$ $1,2,3 \ldots, q, i=1,2,3 \ldots, r), \mathrm{r}$ is the fuzzy rules and $M_{l i}$ is the fuzzy set, and $x_{i}(t)(i=1,2,3 \ldots, r)$ is a prevariable, and in this model it is also a prevariable of coronary artery system, $A_{i}$ and $B_{i}$ are the given system state matrices, $E \cos (t)$ is the periodic stimulus as defined by medicine, and $h(t)$ is a timevarying delay satisfying

$$
\begin{aligned}
& 0 \leq h(t) \leq \tau, \\
& 0 \leq \dot{h}(t) \leq \mu \leq 1
\end{aligned}
$$

As described above, the fuzzy response system is described as follows.

Rule I

$$
\begin{aligned}
& \text { IF } \widehat{x}_{1}(t) \text { is } M_{l 1} \\
& \text { THEN } \dot{x}_{s}(t)=\left(A_{1}+\Delta A\right) x_{m}(t) \\
& \quad+\left(B_{1}+\Delta B\right) x_{m}(t-h(t))+E \cos (t)+u(t)
\end{aligned}
$$

Rule II

$$
\begin{aligned}
& \text { IF } \widehat{x}_{2}(t) \text { is } M_{l 2} \\
& \text { THEN } \dot{x}_{s}(t)=\left(A_{2}+\Delta A\right) x_{m}(t) \\
& \quad+\left(B_{2}+\Delta B\right) x_{m}(t-h(t))+E \cos (t)+u(t)
\end{aligned}
$$

Rule $i$

$$
\begin{aligned}
& \text { IF } \widehat{x}_{i}(t) \text { is } M_{l i} \\
& \text { THEN } \dot{x}_{s}(t)=\left(A_{i}+\Delta A\right) x_{m}(t) \\
& \quad+\left(B_{i}+\Delta B\right) x_{m}(t-h(t)) E \cos (t)+u(t)
\end{aligned}
$$

where $x_{s}(t)$ is a state vector for response system, $\widehat{x}_{i}(t)(i=$ $1,2,3 \ldots, r)$ is a prevariable, and in this model it is also a prevariable of coronary artery system and $u(t)$ is a fuzzy adaptive PDC controller.

The master-slave system has the same rule number, state matrix, and fuzzy set.

In order to study the generality of the stabilization conditions of coronary artery system, we generalize the number of fuzzy rules and obtain a more representative T-S Fuzzy coronary artery system (TSFCAS) model, which has $r$ fuzzy rules. The system is described as follows. 
The master system of TSFCAS is described as follows: for rule i in $r$ fuzzy rules,

$$
\begin{aligned}
& \text { IF } x_{i}(t) \text { is } M_{l i} \\
& \text { THEN } \dot{x}_{m}(t)=\left(A_{i}+\Delta A\right) x_{m}(t) \\
& \quad+\left(B_{i}+\Delta B\right) x_{m}(t-h(t))+E \cos (t)
\end{aligned}
$$

the slave system of TSFCAS is described as follows: for rule i in $r$ fuzzy rules:

$$
\begin{aligned}
& \text { IF } x_{i}(t) \text { is } M_{l i} \\
& \text { THEN } \dot{x}_{s}(t)=\left(A_{i}+\Delta A\right) x_{s}(t) \\
& \quad+\left(B_{i}+\Delta B\right) x_{s}(t-h(t))+E \cos (t)+u(t)
\end{aligned}
$$

In the above model, $\mathrm{r}$ is the number of fuzzy rules of the system, regulations:

$$
\begin{aligned}
m_{i}\left(x_{i}(t)\right)= & \prod_{l=1}^{q} M_{l i}\left(x_{l}(t)\right), \quad \sum_{i=1}^{r} m_{i}\left(x_{i}(t)\right) \geq 0 \\
h_{i}\left(x_{i}(t)\right)= & \frac{m_{i}\left(x_{i}(t)\right)}{\sum_{i=1}^{r} m_{i}\left(x_{i}(t)\right)}, \\
& h_{i}\left(x_{i}(t)\right) \geq 0, \sum_{i=1}^{r} h_{i}\left(x_{i}(t)\right)=1
\end{aligned}
$$

Then the TSFCAS model is expressed as follows.

The dynamic equation of the master system (driving system) of TSFCAS is as follows:

$$
\begin{aligned}
& \dot{x}_{m}(t)=\sum_{i=1}^{r} h_{i}\left(x_{m i}(t)\right) \\
& \cdot {\left[\left(A_{i}+\Delta A\right) x_{m}(t)+\left(B_{i}+\Delta B\right) x_{m}(t-h(t))\right]+E } \\
& \cdot \cos (t)
\end{aligned}
$$

The dynamic equation of the slave system (response system) of TSFCAS is as follows:

$$
\begin{aligned}
\dot{x}_{s}(t) & =\sum_{i=1}^{r} h_{i}\left(x_{s i}(t)\right) \\
\cdot & {\left[\left(A_{i}+\Delta A\right) x_{s}(t)+\left(B_{i}+\Delta B\right) x_{s}(t-h(t))\right]+E } \\
\cdot & \cos (t)+u(t)
\end{aligned}
$$

Remark 1. The control objective is to design a controller $u(t)$ for the response system, so that the error closed-loop system is composed of the drive system and the response system is stabilized. This paper uses fuzzy PDC controller.

\section{Fuzzy PDC Controller Design of TSFCAS}

In this section, we design a fuzzy PDC controller $u(t)$.

For ease of presentation, definitions,

$$
h_{i}=\sum_{i=1}^{r} h_{i}\left(x_{m i}(t)\right)=\sum_{i=1}^{r} h_{i}\left(x_{s i}(t)\right)
$$

Define the error function as

$$
e(t)=x_{m}(t)-x_{s}(t)
$$

According to (10) and (11), the error system is as follows:

$$
\begin{aligned}
\dot{e}(t)= & \dot{x}_{m}(t)-\dot{x}_{s}(t) \\
= & h_{i}\left[\left(A_{i}+\Delta A\right) e(t)+\left(B_{i}+\Delta B\right) e(t-h(t))\right] \\
& -u(t)
\end{aligned}
$$

For each fuzzy rule of the slave system, the rules of the fuzzy adaptive controller are described as follows:

$$
\begin{gathered}
\text { IF } x_{s i}(t) \text { is } M_{l i} \\
\text { THEN } u(t)=K_{i} e(t)
\end{gathered}
$$

where $K_{i}$ is the gain matrix of the PDC controller [28].

Corresponding to the T-S fuzzy dynamic equation of the system, the fuzzy adaptive controller based on PDC is obtained as follows:

$$
u(t)=h_{i}\left[K_{i} e(t)\right]
$$

The error system of the coronary artery fuzzy closed-loop system is obtained as follows:

$$
\begin{aligned}
\dot{e}(t)= & \dot{x}_{m}(t)-\dot{x}_{s}(t) \\
= & h_{i}\left[\left(A_{i}+\Delta A\right) e(t)+\left(B_{i}+\Delta B\right) e(t-h(t))\right] \\
& -u(t) \\
= & h_{i}\left[\left(A_{i}+\Delta A-K_{i}\right) e(t)+\left(B_{i}+\Delta B\right) e(t-h(t))\right]
\end{aligned}
$$

Definition 2. The uncertainties of the system are described as follows:

$$
\begin{gathered}
\Delta A=D_{a} F_{a}(t) E_{a}, \\
\Delta B=D_{b} F_{b}(t) E_{b}
\end{gathered}
$$

where $D_{a}, E_{a}, D_{b}, E_{b}$ are appropriate dimensional constant matrices, and the unknown matrices $F_{a}(t), F_{b}(t)$ satisfy

$$
\begin{aligned}
& F_{a}^{T}(t) F_{a}(t) \leq I, \\
& F_{b}^{T}(t) F_{b}(t) \leq I
\end{aligned}
$$

Lemma 3 ([27]). For a vector-valued function $x(t) \in \mathfrak{R}^{n}$, and other matrices $H_{1}, H_{2} \in \mathfrak{R}^{n \times n}, Z \in \mathfrak{R}^{2 n \times 2 n}$, and functional $h(t) \geq 0$, and a symmetric positive definite matrix $X \in \mathfrak{R}^{n \times n}$, The following integral inequalities hold:

$$
-\int_{t-h}^{t} \dot{x}^{T}(s) X \dot{x}(s) d s \leq \xi^{T}(t) \Upsilon \xi(t)+h \xi^{T}(t) Z \xi(t)
$$


where

$$
\begin{aligned}
\Upsilon & :=\left[\begin{array}{cc}
H_{1}^{T}+H_{1} & -H_{1}^{T}+H_{2} \\
* & -H_{2}^{T}-H_{2}
\end{array}\right], \\
\xi(t) & :=\left[\begin{array}{c}
x(t) \\
x(t-h)
\end{array}\right], \\
{\left[\begin{array}{cc}
X & Y \\
* & Z
\end{array}\right] } & \geq 0, \\
Y & :=\left[\begin{array}{ll}
H_{1} & H_{2}
\end{array}\right]
\end{aligned}
$$

Lemma 4 ([29]). For the symmetric matrices $F_{i} \in \Re^{n \times n}$, if there exist real scalars $\varepsilon_{i} \geq 0, i=1,2,3 \cdots, p$, such that $F_{0}-$ $\sum_{i=1}^{p} \varepsilon_{i} F_{i}>0$, we can get

$$
\Phi^{T} F_{0} \Phi>0, \quad \forall \Phi \neq 0 \Longrightarrow \Phi^{T} F_{i} \Phi \geq 0
$$

Lemma 5 ([26]). For a given symmetric positive definite matrix $\zeta$, in $\left[n_{1}, n_{2}\right] \longrightarrow \mathfrak{R}^{n}, \varphi$ is differentiable function, and we have

$$
\int_{n_{1}}^{n_{2}} \int_{\theta}^{n_{2}} \dot{\varphi}^{T}(t) \zeta \dot{\varphi}(t) d t d \theta \geq \eta_{1}^{T} \zeta \eta_{1}+2 \eta_{2}^{T} \zeta \eta_{2}
$$

$$
\left(n_{1} \neq n_{2}\right)
$$

where

$$
\begin{aligned}
\eta_{1}= & \frac{2}{\left(n_{2}-n_{1}\right)} \varphi\left(n_{2}\right)-\frac{2}{\left(n_{2}-n_{1}\right)^{2}} \int_{n_{1}}^{n_{2}} \varphi(\theta) d \theta \\
\eta_{2}= & \frac{1}{\left(n_{2}-n_{1}\right)} \varphi\left(n_{2}\right)-\frac{2}{\left(n_{2}-n_{1}\right)^{2}} \int_{n_{1}}^{n_{2}} \varphi(\theta) d \theta \\
& +\frac{6}{\left(n_{2}-n_{1}\right)^{3}} \int_{n_{1}}^{n_{2}} \int_{\theta}^{n_{2}} \varphi(t) d t d \theta
\end{aligned}
$$

\section{Main Result}

In this section, we use the lemma mentioned above to get a new synchronization method.

Theorem 6. Consider coronary artery fuzzy closed-loop error system, for given a positive constant $\tau>0, \varepsilon_{1} \geq 0, \varepsilon_{2} \geq 0$, if there exist positive symmetric matrices $P>0, Q>0, V>0$, $W>0$, and any matrices $\mathrm{H}_{1}, \mathrm{H}_{2}$, satisfying the following LMIs:

$$
\left[\begin{array}{cccccccc}
\Gamma_{1} & \Gamma_{2} & \Gamma_{4} & \Gamma_{5} & 0 & 3 W & A_{i}^{T} V & \tau H_{1}^{T} \\
* & \Gamma_{3} & \frac{\tau^{4}}{4} B_{i}^{T} W D_{a} & \frac{\tau^{4}}{4} B_{i}^{T} W D_{b} & 0 & 0 & B_{i}^{T} V & \tau H_{2}^{T} \\
* & * & -\varepsilon_{1} I+\frac{\tau^{4}}{4} D_{a}^{T} W D_{a} & \frac{\tau^{4}}{4} D_{a}^{T} W D_{b} & 0 & 0 & D_{a}^{T} V & 0 \\
* & * & * & -\varepsilon_{2} I+\frac{\tau^{4}}{4} D_{b}^{T} W D_{b} & 0 & 0 & D_{b}^{T} V & 0 \\
* & * & * & * & -3 W & \frac{6}{\tau} W & 0 & 0 \\
* & * & * & * & * & -\frac{18}{\tau^{2}} W & 0 & 0 \\
* & * & * & * & * & * & -V & 0 \\
* & * & * & * & * & * & * & -V
\end{array}\right]<0,
$$

where

$$
\begin{aligned}
\Gamma_{1}= & P A_{i}+A_{i}^{T} P+P K_{i}+K_{i}^{T} P+Q+H_{1}+H_{1}^{T} \\
& +\varepsilon_{1} E_{a}^{T} E_{a}+\frac{\tau^{4}}{4} A_{i}^{T} W A_{i}-\frac{\tau^{4}}{4} A_{i}^{T} W K_{i} \\
& -\frac{\tau^{4}}{4} K_{i}^{T} W A_{i}+\frac{\tau^{4}}{4} K_{i}^{T} W K_{i}-\frac{3}{2} \tau^{2} W \\
& -A_{i}^{T} V K_{i}-K_{i}^{T} V A_{i}+K_{i}^{T} V K_{i} \\
\Gamma_{2}= & P B_{i}-H_{1}^{T}+H_{2}+\frac{\tau^{4}}{4} A_{i}^{T} W B_{i}-\frac{\tau^{4}}{4} K_{i}^{T} W B_{i} \\
& -K_{i}^{T} V B_{i}
\end{aligned}
$$

$$
\begin{aligned}
& \Gamma_{3}=\varepsilon_{2} E_{b}^{T} E_{b}-(1-\mu) Q-H_{2}^{T}-H_{2}+\frac{\tau^{4}}{4} B_{i}^{T} W B_{i} \\
& \Gamma_{4}=P D_{a}+\frac{\tau^{4}}{4} A_{i}^{T} W D_{a}-\frac{\tau^{4}}{4} K_{i}^{T} W D_{a}-K_{i}^{T} V D_{a} \\
& \Gamma_{5}=P D_{b}+\frac{\tau^{4}}{4} A_{i}^{T} W D_{b}-\frac{\tau^{4}}{4} K_{i}^{T} W D_{b}-K_{i}^{T} V D_{b}
\end{aligned}
$$

Then the closed-loop coronary artery error system will be asymptotically stable.

Proof. Construct the following LKF:

$$
V(t)=\sum_{i=1}^{4} V_{i}(t),
$$


where

$$
\begin{aligned}
& V_{1}(t)=e^{T}(t) P e(t), \\
& V_{2}(t)=\int_{t-h(t)}^{t} e^{T}(s) Q e(s) d s, \\
& V_{3}(t)=\int_{-\tau}^{0} \int_{t+\theta}^{t} \dot{e}^{T}(s) R \dot{e}(s) d s d \theta, \\
& V_{4}(t)=\frac{\tau^{2}}{2} \int_{t-\tau}^{t} \int_{s}^{t} \int_{u}^{t} \dot{e}^{T}(v) W \dot{e}(v) d v d u d s,
\end{aligned}
$$

The time derivative of $V(t)$

$$
\dot{V}(t)=\sum_{i=1}^{4} \dot{V}_{i}(t)
$$

where

$$
\begin{aligned}
& \dot{V}_{1}(t)=\dot{e}^{T}(t) P e(t)+e^{T}(t) P \dot{e}(t)=h_{i}\left[e^{T}(t)\right. \\
& \cdot {\left.\left[P\left(A_{i}+\Delta A-K_{i}\right)+\left(A_{i}+\Delta A-K_{i}\right)^{T} P\right] e(t)\right] } \\
&+ h_{i}\left[2 e^{T}(t) P\left(B_{i}+\Delta B\right) e(t-h(t))\right] \\
& \dot{V}_{2}(t)=h_{i}\left[e^{T}(t) Q e(t)\right]-h_{i}\left[(1-\mu) e^{T}(t-h(t))\right. \\
& \cdotQ e(t-h(t))] \\
& \dot{V}_{3}(t)=\tau \dot{e}^{T}(t) \operatorname{Re}(t)-\int_{t-\tau}^{t} \dot{e}^{T}(s) \operatorname{Re}(s) d s \\
& \dot{V}_{4}(t)=\frac{\tau^{4}}{4} \dot{e}^{T}(t) W \dot{e}(t)-\frac{\tau^{2}}{2} \\
& \cdot \int_{t-\tau}^{t} \int_{s}^{t} \dot{e}^{T}(u) W \dot{e}(u) d u d s
\end{aligned}
$$

For $\dot{V}_{3}(t)$, according to Lemma 3 , we obtain

$$
\begin{aligned}
\dot{V}_{3}(t)= & \tau \dot{e}^{T}(t) \operatorname{Re}(t)-\int_{t-\tau}^{t} \dot{e}^{T}(s) \operatorname{Re}(s) d s \\
\leq & \tau \dot{e}^{T}(t) \operatorname{Re}(t)-\int_{t-h(t)}^{t} \dot{e}^{T}(s) \operatorname{Re}(s) d s \\
\leq & \tau \dot{e}^{T}(t) \operatorname{Re}(t) \\
& +h_{i}\left[\xi^{T}(t) \Upsilon \xi(t)+\tau \xi^{T}(t) Z \xi(t)\right]
\end{aligned}
$$

where

$$
\begin{gathered}
\Upsilon:=\left[\begin{array}{cc}
H_{1}^{T}+H_{1} & -H_{1}^{T}+H_{2} \\
* & -H_{2}^{T}-H_{2}
\end{array}\right], \\
\xi(t):=\left[\begin{array}{c}
x(t) \\
x(t-h)
\end{array}\right], \\
Z:=\left[\begin{array}{c}
H_{1}^{T} \\
H_{2}^{T}
\end{array}\right], \\
R^{-1}\left[\begin{array}{ll}
H_{1} & H_{2}
\end{array}\right]
\end{gathered}
$$

For $\dot{V}_{4}(t)$, the proposed Lemma 5 was applied as follows:

$$
\frac{\tau^{2}}{2} \eta(t) \geq \phi_{1}^{T}(t) W \phi_{1}(t)+2 \phi_{2}^{T}(t) W \phi_{2}(t)
$$

where

$$
\begin{aligned}
\eta(t)= & \int_{t-\tau}^{t} \int_{s}^{t} \dot{e}^{T}(u) W \dot{e}(u) d u d s \\
\phi_{1}(t)= & \int_{t-\tau}^{t} \int_{s}^{t} \dot{e}(u) d u d s=h e(t)-\int_{t-\tau}^{t} e(s) d s \\
\phi_{2}(t)= & \int_{t-\tau}^{t} \int_{s}^{t} \dot{e}(u) d u d s \\
& -\frac{3}{h} \int_{t-\tau}^{t} \int_{s}^{t} \int_{u}^{t} \dot{e}(v) d v d u d s \\
= & -\frac{h}{2} e(t)-\int_{t-\tau}^{t} e(s) d s+\frac{3}{h} \int_{t-\tau}^{t} \int_{s}^{t} e(u) d u d s
\end{aligned}
$$

Combining (27), (29), (32), and (33), we obtain

$$
\dot{V}(t) \leq h_{i} \psi^{T}(t) \Xi \psi(t)
$$

where

$$
\begin{aligned}
& \psi(t)=\left[\begin{array}{llll}
e^{T}(t) e^{T}(t-h(t)) & \left(F_{a}(t) E_{a} e(t)\right)^{T} & \left(F_{b}(t) E_{b} e(t-h(t))\right)^{T} & \int_{t-\tau}^{t} e^{T}(s) d s \int_{t-\tau}^{t} \int_{s}^{t} e^{T}(u) d u d s
\end{array}\right] \\
& \Xi=\left[\begin{array}{cccccc}
\Lambda_{1} & \Lambda_{2} & \Lambda_{4} & \Lambda_{5} & 0 & 3 W \\
* & \Lambda_{3} & \tau B_{i}^{T} R D_{a}+\frac{\tau^{4}}{4} B_{i}^{T} W D_{a} & \tau B_{i}^{T} R D_{b}+\frac{\tau^{4}}{4} B_{i}^{T} W D_{b} & 0 & 0 \\
* & * & \tau D_{a}^{T} R D_{a}+\frac{\tau^{4}}{4} D_{a}^{T} W D_{a} & \tau D_{a}^{T} R D_{b}+\frac{\tau^{4}}{4} D_{a}^{T} W D_{b} & 0 & 0 \\
* & * & * & \tau D_{b}^{T} R D_{b}+\frac{\tau^{4}}{4} D_{b}^{T} W D_{b} & 0 & 0 \\
* & * & * & * & -3 W & \frac{6}{\tau} W \\
* & * & * & * & * & -\frac{18}{\tau^{2}} W
\end{array}\right]<0,
\end{aligned}
$$


where

$$
\begin{aligned}
\Lambda_{1}= & P A_{i}+A_{i}^{T} P+P K_{i}+K_{i}^{T} P+Q+H_{1}+H_{1}^{T} \\
& +\tau A_{i}^{T} R A_{i}+\tau H_{1}^{T} R^{-1} H_{1}+\frac{\tau^{4}}{4} A_{i}^{T} W A_{i} \\
& -\frac{\tau^{4}}{4} A_{i}^{T} W K_{i}-\frac{\tau^{4}}{4} K_{i}^{T} W A_{i}+\frac{\tau^{4}}{4} K_{i}^{T} W K_{i} \\
& -\frac{3}{2} \tau^{2} W-\tau A_{i}^{T} R K_{i}-\tau K_{i}^{T} R A_{i}+\tau K_{i}^{T} R K_{i} \\
\Lambda_{2}= & P B_{i}-H_{1}^{T}+H_{2}+\tau A_{i}^{T} R B_{i}+\tau H_{1}^{T} R^{-1} H_{2} \\
& +\frac{\tau^{4}}{4} A_{i}^{T} W B_{i}-\frac{\tau^{4}}{4} K_{i}^{T} W B_{i}-\tau K_{i}^{T} R B_{i} \\
\Lambda_{3}= & \tau B_{i}^{T} R B_{i}-(1-\mu) Q-H_{2}^{T}-H_{2}+\tau H_{2}^{T} R^{-1} H_{2} \\
& +\frac{\tau^{4}}{4} B_{i}^{T} W B_{i} \\
\Lambda_{4}= & P D_{a}+\tau A_{i}^{T} R D_{a}+\frac{\tau^{4}}{4} A_{i}^{T} W D_{a}-\frac{\tau^{4}}{4} K_{i}^{T} W D_{a} \\
& -\tau K_{i}^{T} R D_{a} \\
\Lambda_{5}= & P D_{b}+\tau A_{i}^{T} R D_{b}+\frac{\tau^{4}}{4} A_{i}^{T} W D_{b}-\frac{\tau^{4}}{4} K_{i}^{T} W D_{b} \\
& -\tau K_{i}^{T} R D_{b}
\end{aligned}
$$

If $\Xi<0$, we obtain $\dot{V}(t)<0$. Applying condition (5), we have that

$$
\left(F_{a}(t) E_{a} e(t)\right)^{T}\left(F_{a}(t) E_{a} e(t)\right) \leq e^{T}(t) E_{a}^{T} E_{a} e(t),
$$

and similarly we can get

$$
\begin{gathered}
\left(F_{b}(t) E_{b} e(t-h(t))\right)^{T}\left(F_{b}(t) E_{b} e(t-h(t))\right) \\
\leq e^{T}(t-h(t)) E_{b}^{T} E_{b} e(t-h(t)),
\end{gathered}
$$

and by using Lemma 4 , we can define

$$
\begin{aligned}
& \varepsilon_{1} \geq 0, \\
& \varepsilon_{2} \geq 0 \text {, } \\
& T_{1}=\operatorname{diag}\left\{\begin{array}{llllll}
E_{a}^{T} E_{a} & 0 & -I & 0 & 0 & 0
\end{array}\right\}, \\
& T_{2}=\operatorname{diag}\left\{\begin{array}{llllll}
0 & E_{a}^{T} E_{a} & -I & 0 & 0 & 0
\end{array}\right\}
\end{aligned}
$$

such that $\Xi+\varepsilon_{1} T_{1}+\varepsilon_{2} T_{2}<0$. Considering the existence of nonlinear terms such as $\tau A_{i}^{T} R A_{i}$ in inequalities, definition $V=\tau R$, applying the well-known Schur complement, we can get LMI (18). This completes the proof.

Remark 7. Systems (12) and (13) are asymptotically synchronized if the error system (15) satisfied $t \longrightarrow \infty, e(t) \longrightarrow 0$. In this paper, we design LKF (29) and we can get the LMI (27) is less than zero by using the MATLAB LMI toolbox, achieving asymptotic stability of the error closed-loop system (15).
Remark 8. Compared with the traditional system model to the problem of tracking control behavior, T-S fuzzy chaotic system model used IF-THEN fuzzy rules to represent the local dynamic characteristics of chaotic systems. The latter part of each fuzzy rule is represented by the linear model of the local region of the state space, and the T$S$ fuzzy model of the nonlinear system is obtained by the integration of the local models. The controller based on T-S fuzzy model can be designed by using the linear system control theory and Lyapunov theory. This is the advantage of using T-S fuzzy chaotic system model in this paper.

\section{Simulation}

In this section, we will give the following numerical examples to demonstrate the effectiveness of the proposed control strategy.

Example 1. According to the follow parameters, we can get the phase portraits of TSFCAS (12), (13).

$$
\begin{aligned}
A_{1} & =\left[\begin{array}{cc}
-0.15 & 1.7 \\
-49.425 & -0.35
\end{array}\right], \\
A_{2} & =\left[\begin{array}{cc}
-0.15 & 1.7 \\
0.575 & -0.35
\end{array}\right], \\
B_{1} & =\left[\begin{array}{cc}
0 & 0 \\
0.01 & 0.01
\end{array}\right], \\
B_{2} & =\left[\begin{array}{cc}
0 & 0 \\
0.01 & 0.01
\end{array}\right], \\
D_{1} & =E_{1}=\left[\begin{array}{cc}
\sqrt{1.6} & 0 \\
0 & \sqrt{0.05}
\end{array}\right], \\
D_{2} & =E_{2}=\left[\begin{array}{cc}
\sqrt{0.1} & 0 \\
0 & \sqrt{0.3}
\end{array}\right], \\
F_{a}(t) & =F_{b}(t)=\left[\begin{array}{cc}
0.5 \sin (t) & -0.5 \cos (t) \\
-0.5 \cos (t) & 0.5 \sin (t)
\end{array}\right]
\end{aligned}
$$

The membership functions of the TSFCAS (12), (13) are selected as

$$
\begin{aligned}
& h_{1}\left(x_{m 1}(t)\right)=1-\frac{x_{m 1}^{2}}{h^{2}}, \\
& h_{2}\left(x_{m 2}(t)\right)=\frac{x_{m 2}^{2}}{h^{2}}
\end{aligned}
$$




$$
\begin{aligned}
& h_{1}\left(x_{s 1}(t)\right)=1-\frac{x_{s 1}^{2}}{h^{2}}, \\
& h_{2}\left(x_{s 2}(t)\right)=\frac{x_{s 2}^{2}}{h^{2}}
\end{aligned}
$$

$$
\left(h^{2}=100\right)
$$

Initial conditions of the TSFCAS (12), (13) are selected as $x_{m}(0)=(0.2,0)^{T}, x_{s}(0)=(-0.1,0.2)^{T}$, and we choose the time-varying delay as $h(t)=0.125 \sin (2 t)$. On the basis of LMI (27), we can gain matrices P, Q, W by using MATLAB LMI toolbox, and we can obtain the gain matrices $K_{1}, K_{2}$ in controller (18) by using MATLAB LMI toolbox.

$$
\begin{aligned}
P & =\left[\begin{array}{cc}
97.9946 & 0.0000 \\
0.0000 & 97.9946
\end{array}\right], \\
Q & =\left[\begin{array}{cc}
114.8543 & 0.00704 \\
0.0070 & 120.8465
\end{array}\right], \\
W & =\left[\begin{array}{cc}
2.4879 & -0.0005 \\
-0.0005 & 2.6188
\end{array}\right], \\
K_{1} & =\left[\begin{array}{cc}
-0.2473 & -0.0194 \\
-0.0224 & 0.4494
\end{array}\right], \\
K_{2} & =\left[\begin{array}{ll}
1.1317 & 0.3703 \\
0.2518 & 0.4494
\end{array}\right]
\end{aligned}
$$

where Figures 1(a) and 2(a) show the phase diagram of coronary drive chaotic system, which can observe that CAS showed obvious chaotic behavior. Figure 1(b) shows the phase diagram of coronary response chaotic system without controller $u(t)$ and Figure 2(b) shows the phase diagram of coronary response chaotic system with controller $u(t)$. With controller $u(t)$, we can see that the trajectory of coronary response chaotic system (Figure 2(b)) synchronization of the trajectory of coronary drives chaotic system Figure 1(a). The error plots between systems (12) and (13) with different initial conditions and without controller $u(t)$ can be shown in Figure 1(c). Figure 2(d) plots the input of fuzzy PDC controller. Through the PDC controller of fuzzy model $u_{1}(t)$ and $u_{2}(t)$ (Figure $2(\mathrm{~d})$ ), we can see that the error plots between systems (12) and (13) tend to zero in Figure 2(c). The simulation result indicated, this paper proposed the method is correct and effective and has certain practical significance.

Example 2. According to the following parameters, we can get the phase portraits of TSFCAS (12), (13).

$$
\begin{aligned}
A_{1} & =\left[\begin{array}{cc}
-0.1 & 2.2 \\
-30.575 & -0.3
\end{array}\right], \\
A_{2} & =\left[\begin{array}{cc}
-0.1 & 2.2 \\
0.575 & -0.35
\end{array}\right], \\
B_{1} & =\left[\begin{array}{cc}
0 & 0 \\
0.02 & 0.02
\end{array}\right], \\
B_{2} & =\left[\begin{array}{cc}
0 & 0 \\
0.02 & 0.02
\end{array}\right], \\
D_{1} & =E_{1}=\left[\begin{array}{cc}
\sqrt{1.8} & 0 \\
0 & \sqrt{0.05}
\end{array}\right], \\
D_{2} & =E_{2}=\left[\begin{array}{cc}
\sqrt{0.3} & 0 \\
0 & \sqrt{0.2}
\end{array}\right], \\
F_{a}(t) & =F_{b}(t)=\left[\begin{array}{cc}
0.5 \sin (t) & -0.5 \cos (t) \\
-0.5 \cos (t) & 0.5 \sin (t)
\end{array}\right]
\end{aligned}
$$

The membership functions and initial conditions of the TSFCAS (12), (13) and the time-varying delay $h(t)$ are the same as Example 1. On the basis of LMI (27), we can gain matrices $\mathrm{P}, \mathrm{Q}, \mathrm{W}$ by using MATLAB LMI toolbox, and we can obtain the gain matrices $K_{1}, K_{2}$ in controller (18) by using MATLAB LMI toolbox.

$$
\begin{aligned}
P & =\left[\begin{array}{ll}
96.3937 & 0.0000 \\
0.0000 & 96.3937
\end{array}\right], \\
Q & =\left[\begin{array}{ll}
135.2740 & -0.0091 \\
-0.0091 & 149.9252
\end{array}\right], \\
W & =\left[\begin{array}{cc}
2.3533 & -0.0032 \\
-0.0032 & 2.5900
\end{array}\right], \\
K_{1} & =\left[\begin{array}{ll}
-0.2779 & -0.0541 \\
-0.0455 & 0.1406
\end{array}\right], \\
K_{2} & =\left[\begin{array}{ll}
1.1854 & 0.4122 \\
0.3389 & 0.1344
\end{array}\right]
\end{aligned}
$$

where Figure 3(a) and Figure 3(b) show the phase diagram of coronary drive chaotic system, which can observe that CAS showed obvious chaotic behavior. Figure 1(b) shows the phase diagram of coronary response chaotic system without controller $u(t)$ and Figure 4(b) shows the phase diagram of coronary response chaotic system with controller $u(t)$. With controller $u(t)$, we can see the trajectory of coronary response chaotic system (Figure 4(b)) synchronization of the trajectory of coronary drive chaotic system Figure 1(a). The error plots between systems (12) and (13) with different initial conditions and without controller $u(t)$ can be shown 


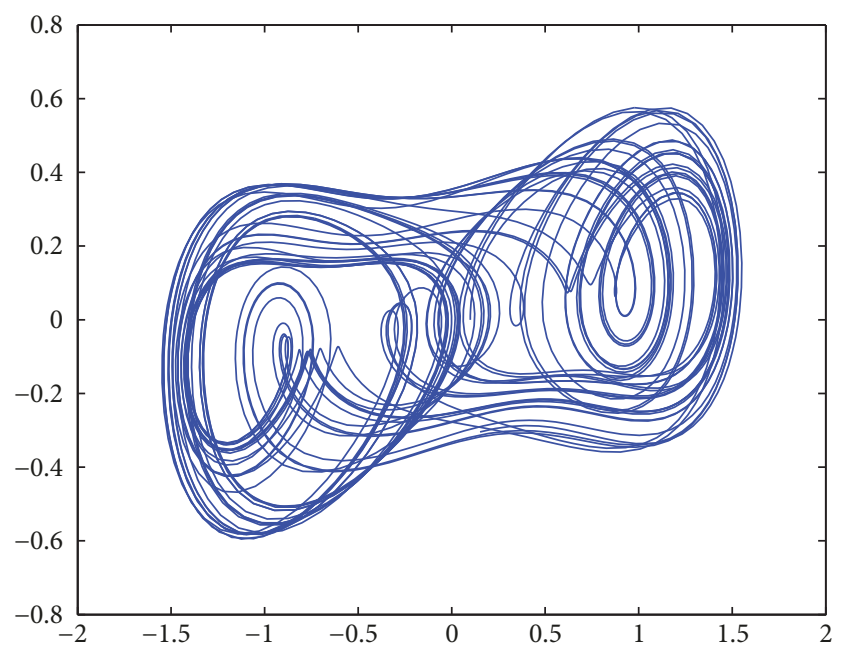

(a) Phase portrait of the master system

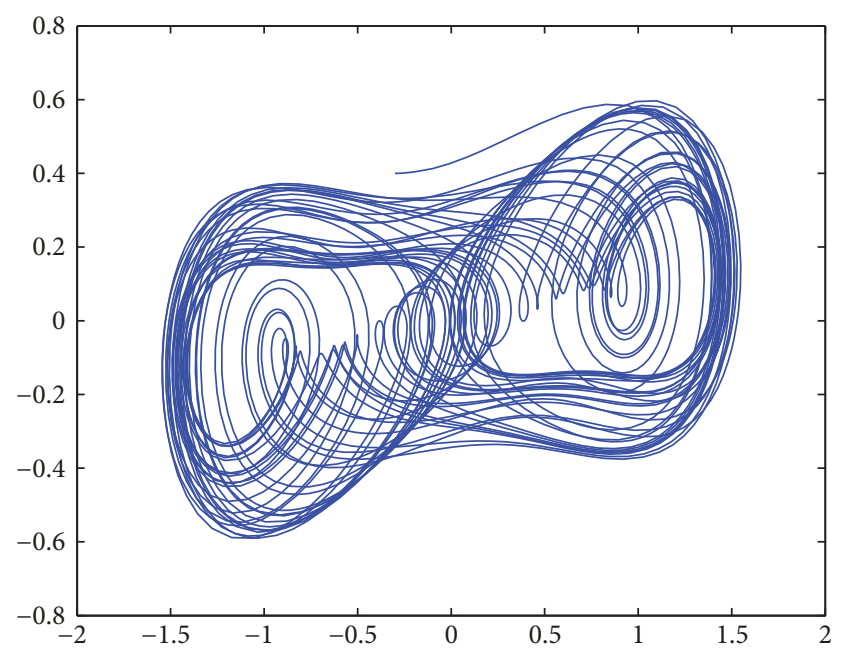

(b) Phase portrait of the slave system

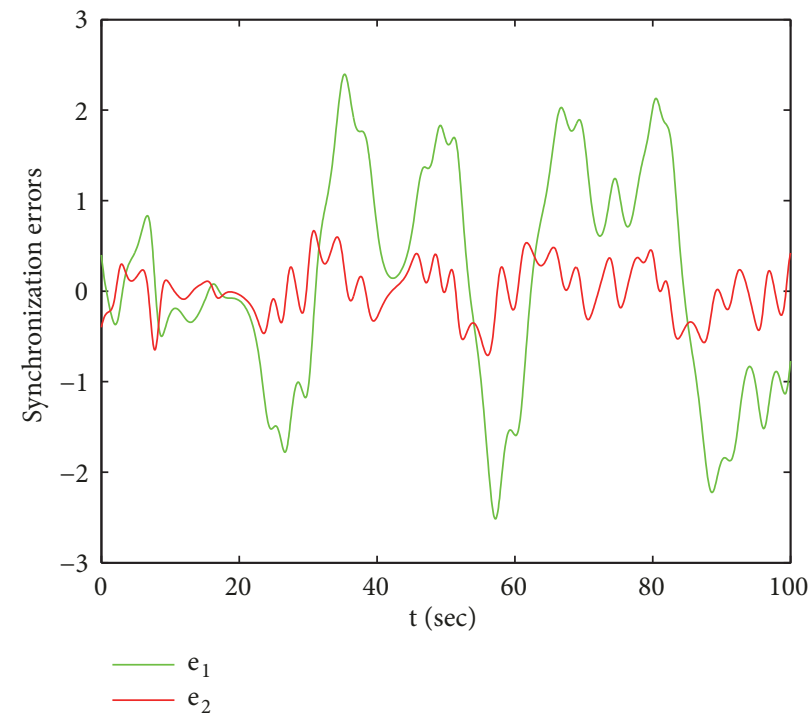

(c) Synchronization errors between the master and the slave systems

FIGURE 1: Behavior of the master-slave systems without control signal.

in Figure 3(c). Figure 4(d) plots the input of fuzzy PDC controller. Through the PDC controller of fuzzy model $u_{1}(t)$ and $u_{2}(t)$ (Figure $4(\mathrm{~d})$ ), we can see that the error plots between systems (12) and (13) tend to zero in Figure 4(c). The simulation result indicated, this paper proposed the method is correct and effective and has certain practical significance. When given different scaling factor, we can see the difference between Examples 1 and 2, such that comparing the error plots between systems (12) and (13) tends to zero time; we can see Figure 2(c) is faster than Figure 4(c), and control signal $u(t)$ control time in Figure 4(c) also needs a little longer.

In the biological and medical sense, by using a synchronization controller to synchronize the chaotic motion state of the diseased vessels to that of the normal vessels, so as to achieve the goal of treatment, it can be seen that the study of coronary vascular chaos synchronization helps to better prevent and treat myocardial infarction, angina pectoris, and other diseases that seriously endanger human health. The CAS is actually a complex nonlinear system and is subject to interference by external factors. For such complex nonlinear systems with uncertain parameters, the T-S fuzzy model can be considered for more precise description. The T-S fuzzy model can well realize the linearization of nonlinear systems and can play an effective role in controller design. Using the T-S fuzzy model, a chaotic system can be regarded as the weighted sum of multiple local linear models, and then the parallel distributed compensation method is used to design the controller, 


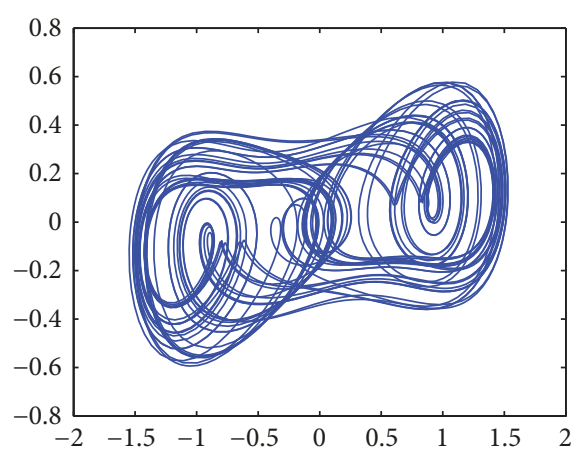

(a) Phase portrait of the master system

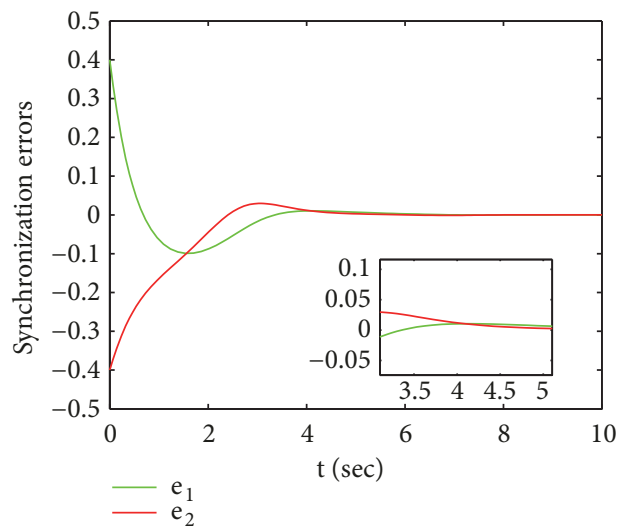

(c) Synchronization errors between the master and the slave systems

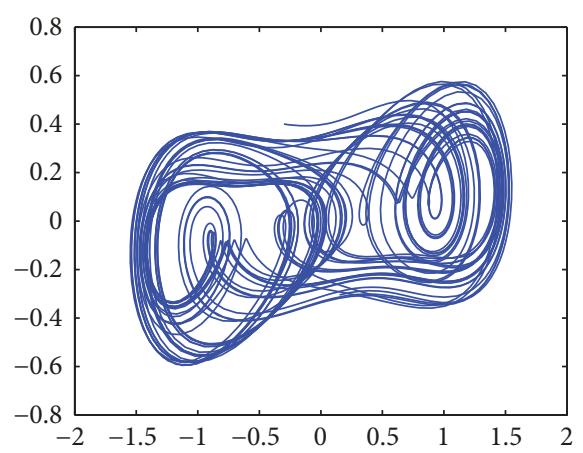

(b) Phase portrait of the slave system

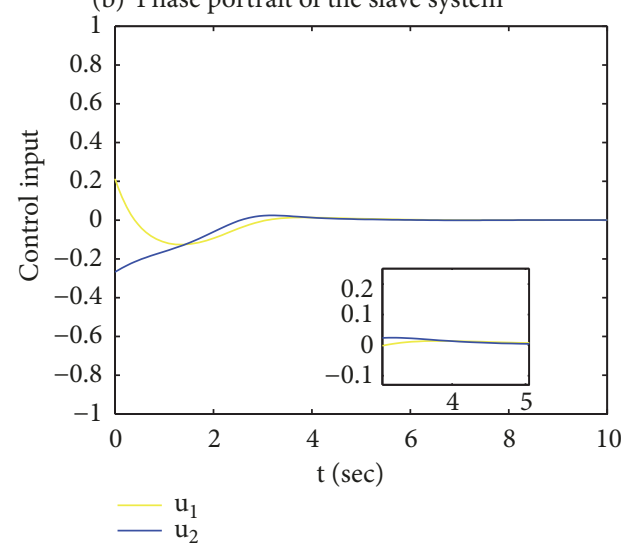

(d) Control input signal $u_{1}(t), u_{2}(t)$

Figure 2: Behavior of the master-slave systems with control signal.

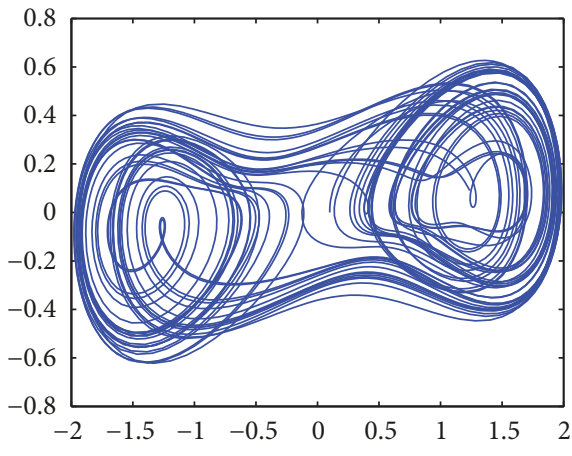

(a) Phase portrait of the master system

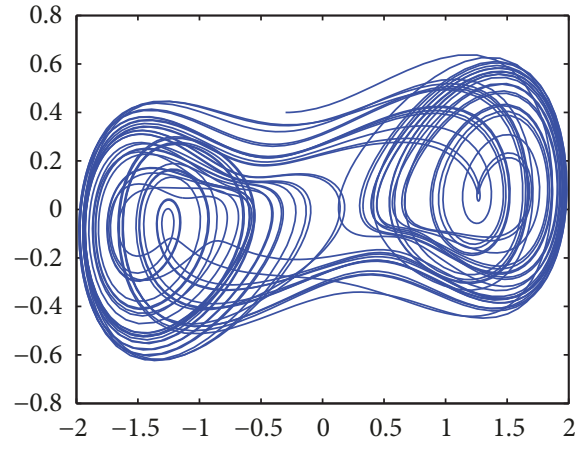

(b) Phase portrait of the slave system

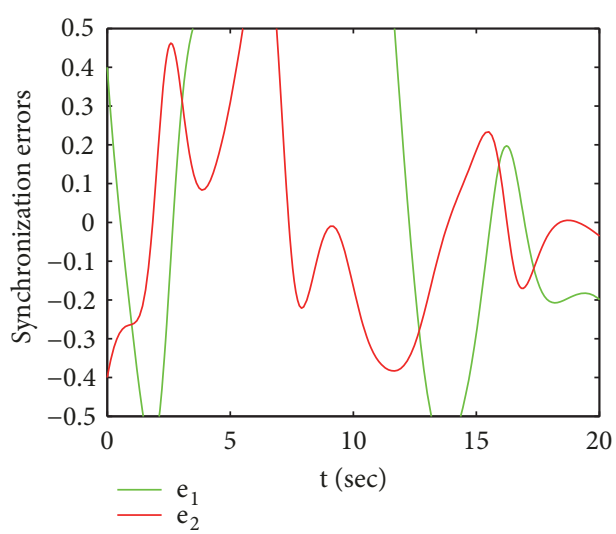

(c) Synchronization errors between the master and the slave systems

FIGURE 3: Behavior of the master-slave systems without control signal. 


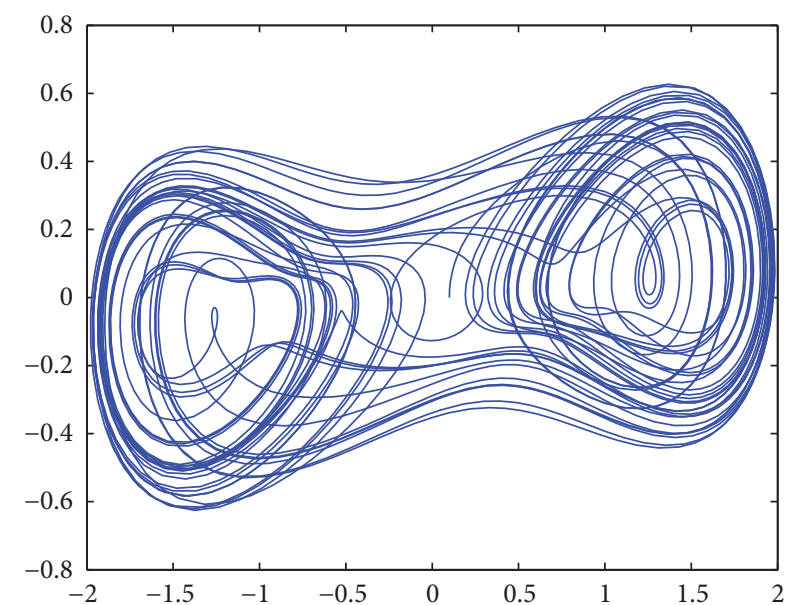

(a) Phase portrait of the master system

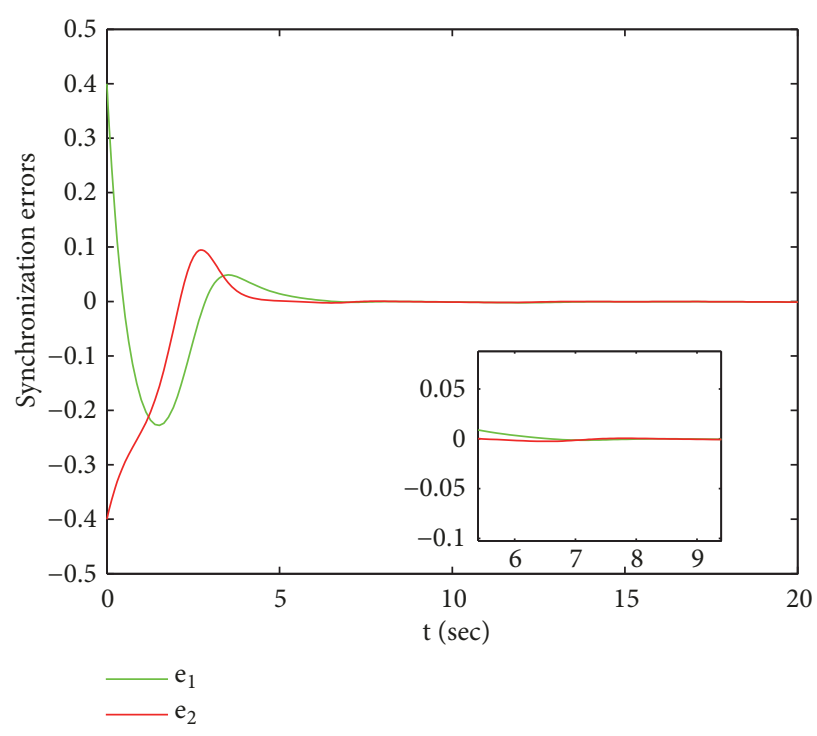

(c) Synchronization errors between the master and the slave systems

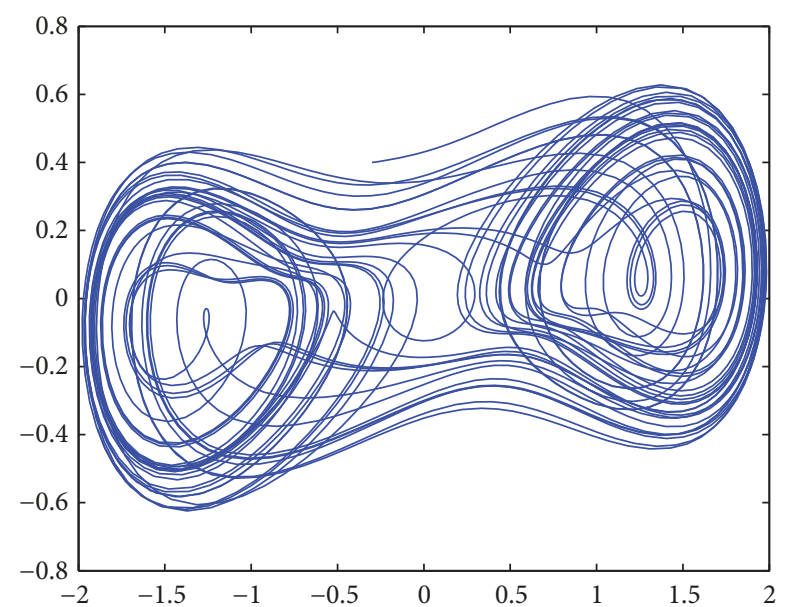

(b) Phase portrait of the slave system

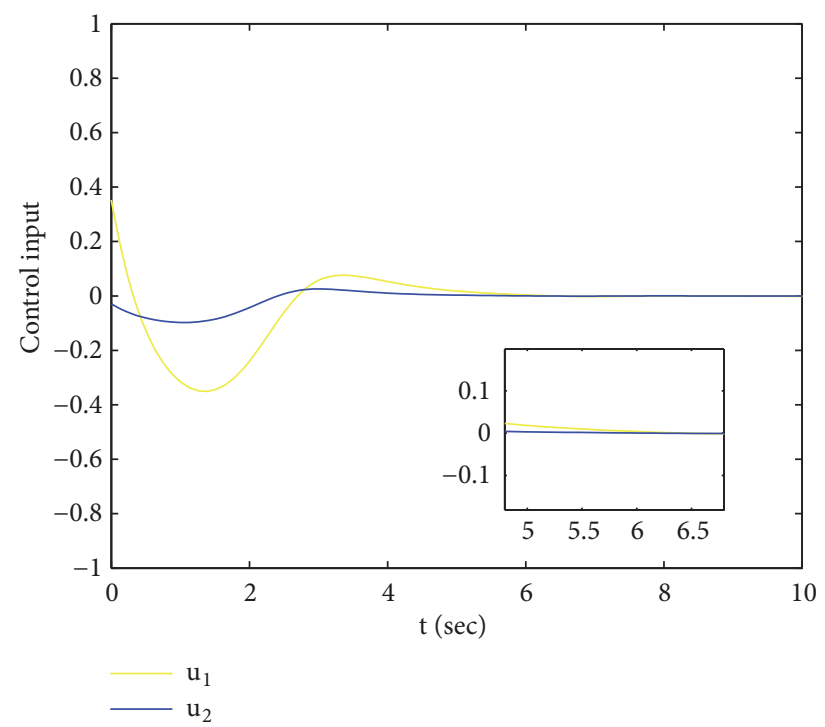

(d) Control input signal $u_{1}(t), u_{2}(t)$

FIGURE 4: Behavior of the master-slave systems with control signal.

thus effectively solving the chaotic synchronization control problem.

\section{Conclusions}

This paper has studied based on the T-S fuzzy model indefinite coronary artery system chaos synchronization control issue. By constructing the uncertain dynamic equation of the coronary artery system, a PDC controller whose fuzzy rule corresponds to the fuzzy model is designed. By using Wirtinger-based double inequality and an improved Moon et al's inequality to construct the appropriate Lyapunov functional, the conditions for system stability in LMI form are derived. Simulation results show that the method proposed in this paper is correct and effective and has certain practical significance. The stability criterion proposed in this paper reduces the conservativeness to a certain extent, but there is still a lot of space for improvement. In the study of CAS, the system is frequently complex and uncertain. Due to the fact that the membership degree of traditional fuzzy sets is two-dimensional, it is very difficult to describe that different objects belong to different fuzzy sets, especially for highly uncertain systems, to improve the system's ability to handle uncertainty. While the membership functions of type-2 fuzzy sets are three-dimensional, it provides the extra degree of freedom the third dimension. In highly uncertain systems, type-2 fuzzy sets are always better than traditional fuzzy sets. Therefore, the application of type- 2 fuzzy model to more complex CAS will be an important research topic in the future. 


\section{Data Availability}

The data used to support the findings of this study are available from the corresponding author upon request.

\section{Conflicts of Interest}

The authors declare that they have no conflicts of interest.

\section{Acknowledgments}

This work is supported in part by the Natural Science Foundation of Tianjin City (18JCYBJC88100 and 15JCYBJC16100) and the Natural Science Foundation of China (61503280).

\section{References}

[1] L. M. Pecora and T. L. Carroll, "Synchronization in chaotic systems," Controlling Chaos, vol. 6, no. 8, pp. 142-145, 1990.

[2] J. Liu, L. Wu, C. Wu, W. Luo, and L. G. Franquelo, "Eventtriggering dissipative control of switched stochastic systems via sliding mode," Automatica, vol. 103, pp. 261-273, 2019.

[3] H. Li, P. Shi, D. Yao, and L. Wu, "Observer-based adaptive sliding mode control for nonlinear Markovian jump systems," Automatica, vol. 64, pp. 133-142, 2016.

[4] J. Liu, S. Laghrouche, and M. Wack, "Observer-based higher order sliding mode control of power factor in three-phase AC/DC converter for hybrid electric vehicle applications," International Journal of Control, vol. 87, no. 6, pp. 1117-1130, 2014.

[5] J.-L. Wang, H.-N. Wu, T. Huang, and M. Xu, "Output synchronization in coupled neural networks with and without external disturbances," IEEE Transactions on Control of Network Systems, vol. 5, no. 4, pp. 2049-2061, 2017.

[6] J. Liu, C. Wu, Z. Wang, and L. Wu, "Reliable filter design for sensor networks using type-2 fuzzy framework," IEEE Transactions on Industrial Informatics, vol. 13, no. 4, pp. 17421752, 2017.

[7] C. Tang, A. Li, and X. Li, "Asymmetric game: a silver bullet to weighted vertex cover of networks," IEEE Transactions on Cybernetics, vol. 48, no. 10, pp. 2994-3005, 2018.

[8] J.-L. Wang, Z. Qin, H.-N. Wu, T. Huang, and P.-C. Wei, "Analysis and pinning control for output synchronization and $\mathrm{H} 1$ output synchronization of multiweighted complex networks," IEEE Transactions on Cybernetics, vol. 49, no. 4, pp. 1314-1326, 2018.

[9] J.-L. Wang, M. Xu, H.-N. Wu, and T. Huang, "Finite-time passivity of coupled neural networks with multiple weights," IEEE Transactions on Cybernetics, pp. 1-13, 2018.

[10] G. Sun, S. Xu, and Z. Li, "Finite-time fuzzy sampled-data control for nonlinear flexible spacecraft with stochastic actuator failures," IEEE Transactions on Industrial Electronics, vol. 64, no. 5, pp. 3851-3861, 2017.

[11] M. Hui, L. Hongjing, Z. Qi et al., "Adaptive dynamic surface control design for uncertain nonlinear strict-feedback systemswith unknown control direction and disturbances," IEEE Transactions on Systems, Man, and Cybernetics: Systems, pp. 110, 2018.

[12] Y. Zhang, J. Sun, H. Liang, and H. Li, "Event-triggered adaptive tracking control for multiagent systems with unknown disturbances," IEEE Transactions on Cybernetics, pp. 1-12, 2018.

[13] S. Mobayen and J. Ma, "Robust finite-time composite nonlinear feedback control for synchronization of uncertain chaotic systems with nonlinearity and time-delay," Chaos, Solitons \& Fractals, vol. 114, pp. 46-54, 2018.

[14] Y. Wu, W. Li, and J. Feng, "Stabilisation of stochastic coupled systems via feedback control based on discrete-time state observations," International Journal of Systems Science, vol. 48, no. 3, pp. 1-10, 2017.

[15] Y. Zhao, J. Wang, F. Yan, and Y. Shen, "Adaptive sliding mode fault-tolerant control for type-2 fuzzy systems with distributed delays," Information Sciences, vol. 473, pp. 227-238, 2019.

[16] F. Nian, X. Liu, and Y. Zhang, "Sliding mode synchronization of fractional-order complex chaotic system with parametric and external disturbances," Chaos, Solitons \& Fractals, vol. 116, pp. 22-28, 2018.

[17] Y. Wang and H. Yu, "Fuzzy synchronization of chaotic systems via intermittent control," Chaos, Solitons \& Fractals, vol. 106, pp. 154-160, 2018.

[18] Z.-G. Wu, P. Shi, H. Y. Su, and J. Chu, "Sampled-data fuzzy control of chaotic systems based on a T-S fuzzy model," IEEE Transactions on Fuzzy Systems, vol. 22, no. 1, pp. 153-163, 2014.

[19] Y. Liu, J. H. Park, B.-Z. Guo, and Y. Shu, "Further results on stabilization of chaotic systems based on fuzzy memory sampled-data control," IEEE Transactions on Fuzzy Systems, vol. 99, pp. 1040-1045, 2017.

[20] X. Su, F. Xia, J. Liu, and L. Wu, "Event-triggered fuzzy control of nonlinear systems with its application to inverted pendulum systems," Automatica, vol. 94, pp. 236-248, 2018.

[21] C. Gong, Y. Li, and X. Sun, "Backstepping control of synchronization for biomathematical model of muscular blood vessel," Journal of Applied Sciences, vol. 24, no. 6, pp. 604-607, 2006.

[22] C. J. Lin, S. K. Yang, and H. T. Yau, "Chaos suppression control of a coronary artery system with uncertainties by using variable structure control," Computers \& Mathematics with Applications, vol. 64, no. 5, p. 64, 2012.

[23] Z. Zhao, J. Zhang, G. Ding, and D. Zhang, "Chaos synchronization of coronary artery system based on higher order sliding mode adaptive control," Acta Physica Sinica, vol. 64, no. 21, pp. 264-271, 2015.

[24] A. Seuret and F. Gouaisbaut, "Wirtinger-based integral inequality: application to time-delay systems," Automatica, vol. 49, no. 9, pp. 2860-2866, 2013.

[25] M. H. Zaheer, M. Rehan, G. Mustafa, and M. Ashraf, "Delayrange-dependent chaos synchronization approach under varying time-lags and delayed nonlinear coupling," ISA Transactions, vol. 53, no. 6, pp. 1716-1730, 2014.

[26] M. Park, O. Kwon, J. H. Park, S. Lee, and E. Cha, "Stability of time-delay systems via Wirtinger-based double integral inequality," Automatica, vol. 55, pp. 204-208, 2015.

[27] X. Zhang, M. Wu, J. She, and Y. He, "Delay-dependent stabilization of linear systems with time-varying state and input delays," Automatica, vol. 41, no. 8, pp. 1405-1412, 2005.

[28] M. Seidi and A. H. D. Markazi, "Model-based fuzzy control of flexible joint manipulator: a LMI approach," in Proceedings of the 5th International Symposium on Mechatronics and its Applications, ISMA 2008, IEEE, Amman, Jordan, May 2008.

[29] Q.-L. Han, "Absolute stability of time-delay systems with sectorbounded nonlinearity," Automatica, vol. 41, no. 12, pp. 2171$2176,2005$. 


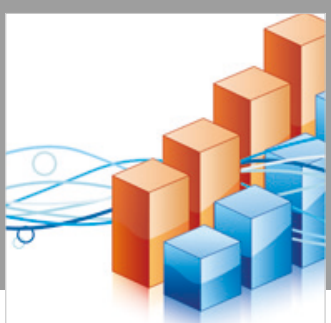

Advances in

Operations Research

\section{-n-m}
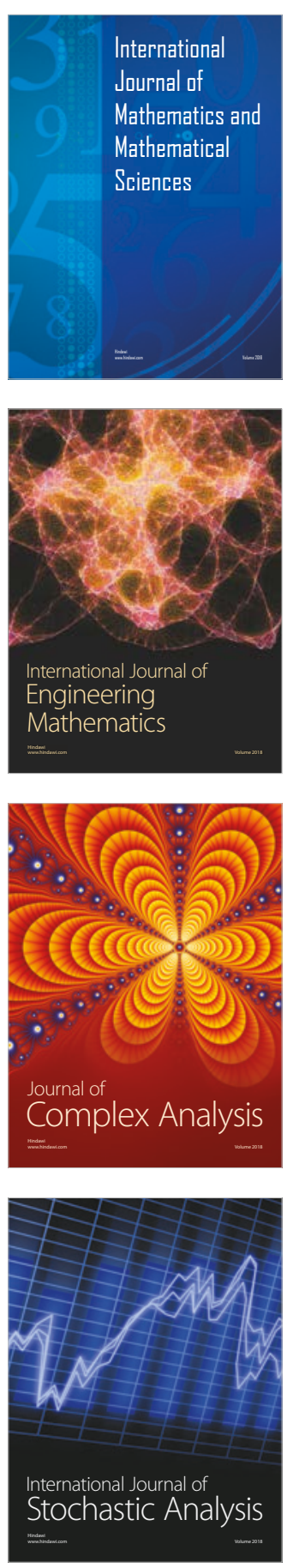
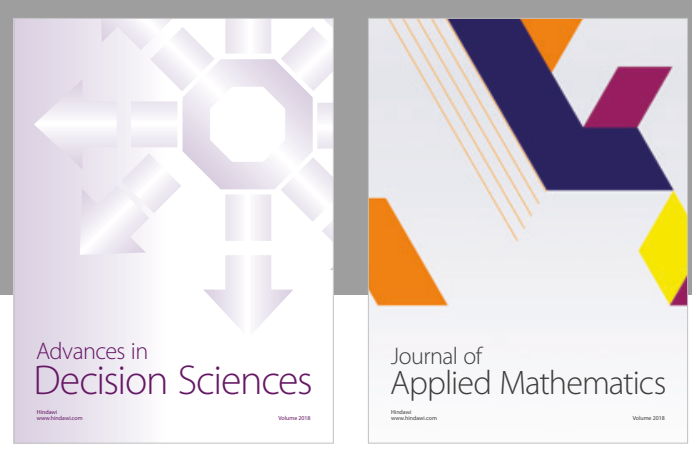

Journal of

Applied Mathematics
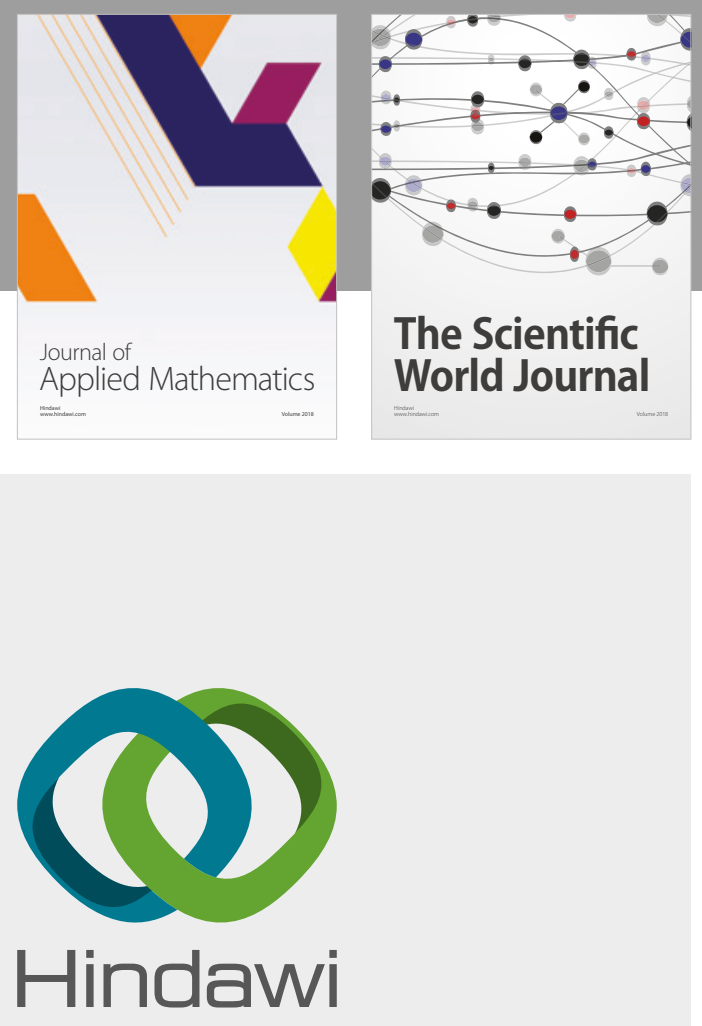

Submit your manuscripts at

www.hindawi.com

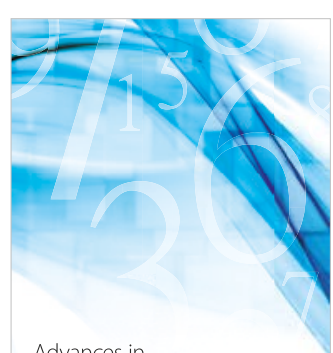

Advances in
Numerical Analysis
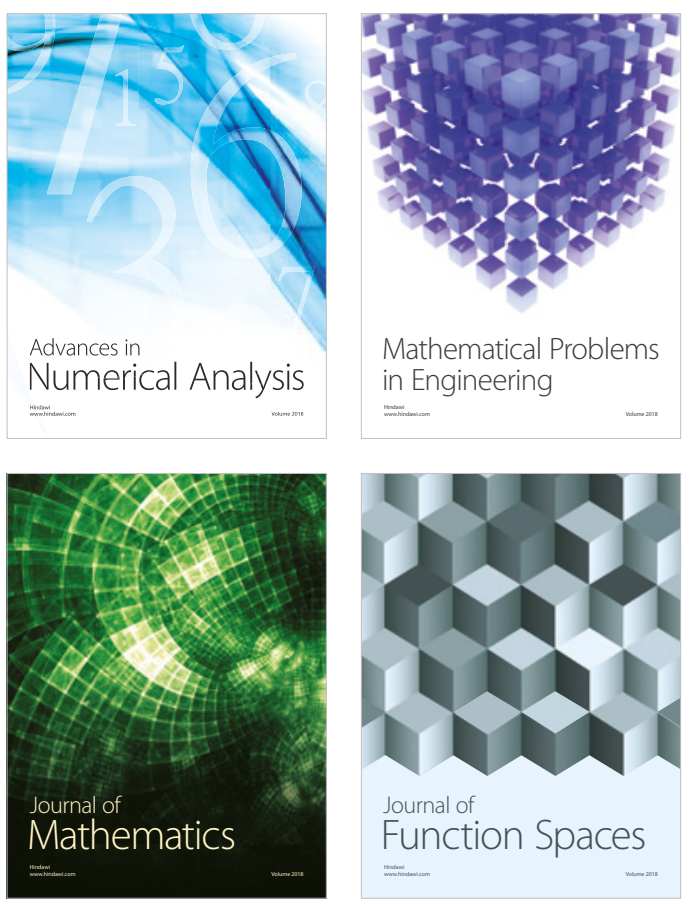

Mathematical Problems in Engineering

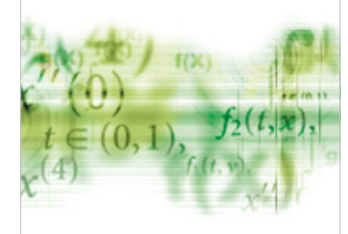

International Journal of

Differential Equations

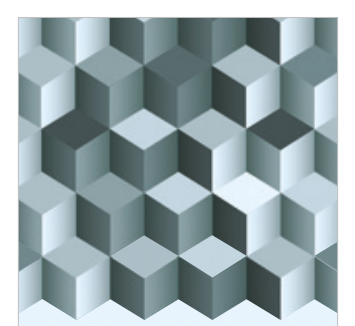

Journal of

Function Spaces
The Scientific

World Journal

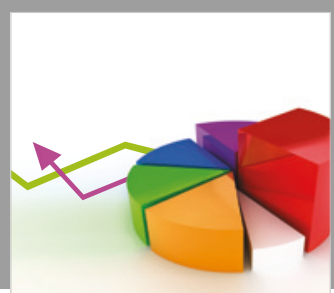

Journal of

Probability and Statistics
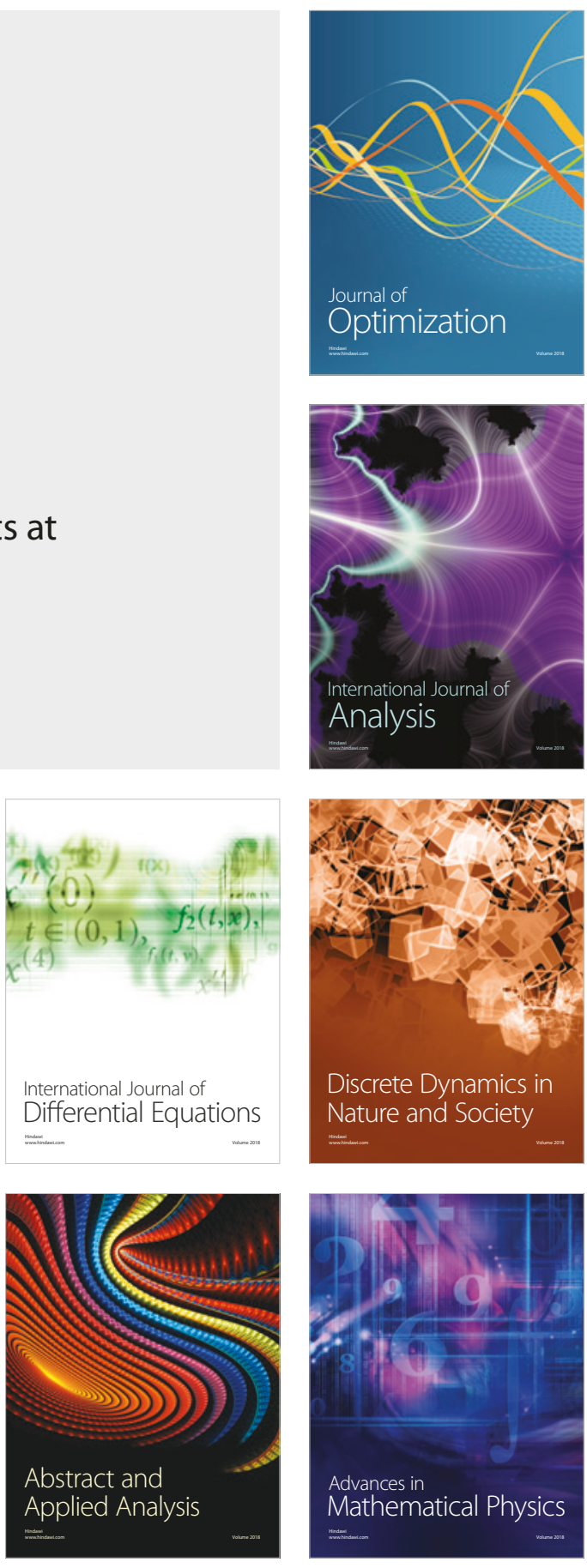